\title{
Plasma-Calprotectin as an Indicator for Immediate Need of Intensive Care Treatment in Suspected Sepsis
}

\section{Asa Emilia Parke ( $\square$ asa.emilia.parke@ki.se )}

Karolinska Institute Department of Medicine: Karolinska Institutet Institutionen for medicin Huddinge https://orcid.org/0000-0002-2133-5323

Christian Unge

KI MedH: Karolinska Institutet Institutionen for medicin Huddinge

\section{David Yu}

Karolinska Institute Department of Laboratory Medicine: Karolinska institutet Institutionen for laboratoriemedicin

\section{Jonas Sunden-Cullberg}

Karolinska Institute Department of Medicine: Karolinska Institutet Institutionen for medicin Huddinge Kristoffer Stralin

Karolinska Institute Department of Medicine: Karolinska Institutet Institutionen for medicin Huddinge

\section{Research}

Keywords: P-calprotectin, predictor, sepsis alert, intensive care, SOFA

Posted Date: March 1st, 2021

DOl: https://doi.org/10.21203/rs.3.rs-213752/v1

License: (c) (i) This work is licensed under a Creative Commons Attribution 4.0 International License. Read Full License 


\section{Abstract}

Introduction: Decisions regarding need of transfer to intensive care of patients with sepsis in the emergency department is challenging. We hypothesised that the new biomarker plasma-calprotectin could be used to help select patients who need intensive care, since it already has shown to be a promising tool in the intensive care unit.

Methods: This prospective study was performed on consecutive sepsis alert patients. The alert summons a multidisciplinary team of physicians from the emergency department, the Department of Infectious Diseases, and the intensive care unit, who evaluate patients for possible infection and decide where to transfer the patient. Blood sampling was performed on consecutive sepsis alert patients. C-reactive protein, procalcitonin, neutrophils, and lymphocytes were routinely analysed, p-calprotectin was analysed from frozen plasma samples using a specific turbidimetric assay.

Results: Among 367 sepsis alert patients, 335 had an infection of whom 66 were immediately transferred to the intensive care unit or high dependency unit. 269 patients were transferred to ordinary wards.

Median p-calprotectin for all infected patients was 2.2 (IQR 1.2-3.9), 3.3 (IQR 1.6-5.2) among those transferred immediately to intensive care unit/ high dependency unit and 2.1 (IQR 1.1-3.5) among those transferred to wards $(p=0.0001)$.

Analysis of area under the receiver-operating characteristic (ROC) curve for transferral to higher care level showed superiority for p-calprotectin compared to procalcitonin and neutrophil-lymphocyte-ratio, both regarding all sepsis alert cases and regarding the patients with infection ( $p<0.001$ for all comparisons)). The best p-calprotectin cut-off $4.0 \mathrm{mg} / \mathrm{L}$ showed sensitivity $42.5 \%$ and specificity $83 \%$ for transferral to ICU/HDU among patients with infection.

Conclusion: In sepsis alert patients, p-calprotectin was significantly elevated in patients transferred immediately to intensive care. P-calprotectin was superior to traditional biomarkers as a predictor of need for intensive care.

Trial registration: Not registered, as the sepsis alert was developed as a clinical supportive tool.

\section{Background}

Sepsis is a frequent reason for hospital care and a leading cause of death, with a mortality ranging from $12-20 \%$, in the western world (1-4). According to a study from Rudd et al, sepsis counted for $20 \%$ of all global deaths in 2017 (5). The World Health Organisation has therefore highlighted early identification of sepsis as a global key challenge in order to provide for better treatment and improved survival rates (6).

Early recognition and treatment of sepsis have been shown to improve outcome for patients but is still a challenge for most hospitals $(7,8)$. There is a need for better biomarkers aimed to support clinical decision making at all levels of sepsis care. 
The Surviving Sepsis Campaign include lactate and procalcitonin (PCT) as the only recommended biomarkers for sepsis care.

Lactate has been found to be a useful screening tool for severe infection and procalcitonin can support discontinuation of empirical antibiotic therapy (9). PCT as well as C-reactive protein (CRP) have diagnostic properties and are tools to distinguish infectious from non-infectious conditions. The prognostic properties of these two biomarkers have been extensively evaluated, but no clear association between them and disease severity (or outcome) has been established (10).

In the early course of sepsis, neutrophils are often activated and elevated and lymphocytes are often decreased (11). Thus, neutrophil-lymphocyte ratio (NLR) has been identified as a useful diagnostic marker of sepsis and has also been shown to have prognostic properties $(12,13)$.

Calprotectin is a protein presented in the cytoplasm of neutrophils and is also expressed on the membrane of monocytes. During activation of neutrophil granulocytes, calprotectin is released into the circulation. Calprotectin has been found to increase in the bloodstream within hours in response to bacteria or endotoxin (14). Thus, calprotectin in blood samples (plasma or serum) has been proposed as a potential biomarker of sepsis (15-20). Recently Bartakova et al found similar performance of PCT, CRP, and NLR for detection of bacterial sepsis in a hospitalized cohort, whereas calprotectin was found to have superior performance (18). Among patients treated in intensive care unit (ICU), another study found calprotectin to be superior to PCT for detection of sepsis and for discriminating sepsis from non-septic conditions. With a cut-off of $1.1 \mathrm{mg} / \mathrm{L}$, calprotectin showed sensitivity $80 \%$ and specificity $46 \%$ for 30 -day mortality (20). Calprotectin has also in a very resent study shown to be a promising biomarker for discrimination sever covid-19 from mild (21). These trials motivate further studies of the prognostic usefulness of calprotectin.

Thus, in the present study, we aimed to focus on patients with suspected sepsis in the emergency department (ED) and evaluate if p-calprotectin was superior to PCT, CRP, and NLR in predicting need of direct transferral to ICU or high dependency unit (HDU), as decided by a multidisciplinary team.

\section{Materials And Methods}

\section{Study design}

The study was performed at Karolinska University Hospital Huddinge, Stockholm, Sweden, a tertiary care hospital with approximately 700 beds.

In the hospital's ED, which has 75,000 visits per year, all patients are routinely subjected to triage with the Rapid Emergency Triage and Treatment System (22). Additionally, since September 2017, a sepsis alert has been implemented, triggered when patients show signs of organ dysfunction combined with symptoms of infection, i.e. fever, history of fever, or clinical suspicion of infection, as we have previously described (23).

Signs of organ dysfunction are either one of A or B: 
1. A) at least one of: oxygen saturation below $90 \%$ despite supplemental oxygen administration, respiratory rate greater than 30 per minute, heart rate greater than 130 beats per minute, systolic blood pressure under $90 \mathrm{mmHg}$, or Glasgow Coma Scale below 8; or

2. B) blood lactate greater than $3.2 \mathrm{mmol} / \mathrm{L}$ combined with at least one of: oxygen saturation below $95 \%$ on room air, respiratory rate greater than 25 per minute, heart rate greater than 110 beats per minute, altered mental status, and temperature above $38.5^{\circ} \mathrm{C}$ or below $35^{\circ} \mathrm{C}$.

Patients who trigger the sepsis alert are urgently (within $15 \mathrm{~min}$ ) assessed bedside by a multidisciplinary team of physicians from the ED, the Department of Infectious Diseases, and the ICU, who establish preliminary diagnosis (assumed infection or not assumed infection), optimize clinical care, and decide level of care, i.e. ordinary ward, HDU, or ICU.

The present study was conducted between 27th of September 2017 and 31st of December 2018. Consecutive patients who triggered the sepsis alert were subjected to structured sampling in the ED, including blood samples for CRP, PCT, neutrophils, lymphocytes, platelets, creatinine, and bilirubin. These samples were immediately analysed at the chemistry laboratory at the hospital, according to routine practice. In addition, blood was collected in a PPT ${ }^{\mathrm{T}}$ Plasma Preparation Tube (Becton Dickinson), which was immediately centrifuged for 10 minutes in order to separate plasma from blood cells, and then directly frozen at $-18^{\circ} \mathrm{C}$. After a maximum of 3 week, the samples were moved to $-70^{\circ} \mathrm{C}$. For all patients with written informed consent, the plasma samples were thawed and analysed for $p$-calprotectin, using a specific particle enhanced turbidimetric assay by Gentian Diagnostics AS in Moss, Norway.

\section{Data collection:}

Data regarding demographic characteristics, comorbidity, physiological parameters, clinical, radiological, and microbiological data, and antimicrobial therapy were collected from the patient's electronic records. Admission sequential organ failure assessment (SOFA) score was calculated from the first physiological parameters registered (in the ambulance or ED) and the first blood samples collected in the ED. Baseline SOFA score was determined by using the least pathological SOFA score parameters available within the time window, 7 days-90 days, prior to admission. If baseline parameters were missing, they were assumed to be normal, as in Seymour (24). Admission SOFA score minus baseline SOFA generated a delta-SOFA score.

\section{Definitions of infection and sepsis}

Sepsis alert patients were considered to have a bacterial infection if they received antibiotic therapy for 4 days or until death or until discharge, in line with definitions suggested by the Centre for Disease Control (25). Patients were considered to have viral infection if a viral microorganism was verified by PCR or serology. Patients with bacterial or viral infection according to the definitions above were considered to have sepsis if they had a delta-SOFA score of 2 or more on admission (26). Septic shock was defined according to the Sepsis-3 criteria (26).

\section{Primary outcome measures}


The primary outcome was need of care in the ICU or HDU, which was decided by the multidisciplinary team.

\section{Statistics}

SPSS statistical software (version 24) was used for comparison of proportions, using the chi-square test, and between-group comparisons, using the Mann-Whitney $U$ test. STATA statistical software was used for receiver operating characteristic $(\mathrm{ROC})$ curve analysis and comparison between areas under the ROC curves (AUC). A p-value of $<0.05$ was considered significant.

\section{Results}

\section{Patient Characteristics}

In total, 592 patients triggered the sepsis alert during the study period, of which 367 were included in the study. Three hundred and thirty-five of these patients had an infection and 32 patients had no infection (Fig. 1). Although the 32 patients without infection triggered the sepsis alert, they were ruled out as infectious sepsis patients by the multidisciplinary team. Among 335 patients with infections, 328 had bacterial and 7 had viral infections, including influenza virus $(n=3)$, respiratory syncytial virus $(n=1)$, noro virus $(n=1)$, rhino virus $(n=1)$, and cytomegalo virus infection $(n=1)$. The cytomegalo virus infection was detected by IgM and IgG serology; the other cases by PCR.

The patients with infection are described in Table 1, divided into those with or without direct transferral to ICU/ HDU. The groups had similar distributions of age, comorbidities, sources of infection, and 28-day survival rates. They had also similar rates of sepsis and delta-SOFA score, although septic shock was significantly more common in those with direct transferral to ICU/ HDU. 
Table 1

Characteristics of patients with assumed infection transferred to ICU/ HDU or ward from ER.

\begin{tabular}{|c|c|c|c|}
\hline Characteristic & $\begin{array}{l}\text { Infection to ICU/HDU from } \\
\operatorname{ER}(n=66)\end{array}$ & $\begin{array}{l}\text { Infection to ward from ER } \\
(n=269)\end{array}$ & $\mathbf{p}$ \\
\hline Age, median years (range) & 71.5(18-94) & $74(19-100)$ & NS \\
\hline Gender, \% female & 35 & 41,0 & NS \\
\hline Charlson index (with age) & $5(0-15)$ & $5(0-18)$ & NS \\
\hline \multicolumn{4}{|l|}{ Comorbidity. } \\
\hline Myocardial infarction & $5(7.5)$ & $36(13)$ & \\
\hline Hart failure & $14(21)$ & $46(17)$ & \\
\hline Peripheral vascular disease & $7(10.5)$ & $24(10)$ & \\
\hline Stroke & $11(16.5)$ & $43(16)$ & \\
\hline Dementia & $8(12)$ & $31(11.5)$ & \\
\hline $\begin{array}{l}\text { Chronic obstructive } \\
\text { pulmonary disease }\end{array}$ & $7(10.5)$ & $48(19)$ & \\
\hline Connective tissue disease & $3(4.5)$ & $19(7)$ & \\
\hline Peptic ulcer disease & $4(6)$ & $7(2.5)$ & \\
\hline Liver disease & $5(7.5)$ & $15(5.5)$ & \\
\hline Diabetes mellitus & $16(24)$ & $64(24.5)$ & \\
\hline Hemiplegia & $1(1.5)$ & $11(4)$ & \\
\hline $\begin{array}{l}\text { Moderate to severe kidney } \\
\text { failure }\end{array}$ & $13(19.5)$ & $57(21)$ & \\
\hline Solid tumour & $10(15)$ & $58(21.5)$ & \\
\hline Lymphoma & $4(6)$ & $8(3)$ & \\
\hline Leukaemia & $5(7.5)$ & $14(5)$ & \\
\hline AIDS & $0(0)$ & $0(0)$ & \\
\hline \multicolumn{4}{|l|}{ Source of infection } \\
\hline Respiratory tract & $22(33)$ & $108(40)$ & NS \\
\hline Urogenital & $18(27)$ & $54(39)$ & NS \\
\hline Intra-abdominal & $7(11)$ & $29(11)$ & NS \\
\hline
\end{tabular}

*Data are presented in numbers (\%), unless otherwise stated. 


\begin{tabular}{|c|c|c|c|}
\hline Characteristic & $\begin{array}{l}\text { Infection to ICU/HDU from } \\
\text { ER }(n=66)\end{array}$ & $\begin{array}{l}\text { Infection to ward from ER } \\
(n=269)\end{array}$ & p \\
\hline Skin/joint/soft tissue/bone & $6(9)$ & $35(13)$ & NS \\
\hline Endocarditis & $1(2)$ & $6(2)$ & NS \\
\hline Other & $0(0)$ & $8(3)$ & NS \\
\hline Unknown & $12(18)$ & $27(10)$ & NS \\
\hline Delta-SOFA score & $2.5(0-8)$ & $3(0-10)$ & NS \\
\hline Sepsis & $57(86)$ & $220(82)$ & NS \\
\hline Septic chock & $16(24)$ & $4(1.5)$ & 0.000 \\
\hline $\begin{array}{l}\text { ICU/HDU/dead during } \\
\text { hospital stay }\end{array}$ & $66(100)$ & $19(7)$ & 0.000 \\
\hline Dead within 28 days & $6(9)$ & $24(10)$ & NS \\
\hline Positive blood cultures & $25(38)$ & $87(32)$ & NS \\
\hline $\begin{array}{l}6 \text { most common agent in } \\
\text { blood culture }\end{array}$ & $(n=25)$ & $(n=87)$ & \\
\hline Escherichia coli & $11(44)$ & $25(29)$ & \\
\hline Streptococcus sp. & $4(16)$ & $15(17)$ & \\
\hline Klebsiella sp. & $3(12)$ & $13(15)$ & \\
\hline Pseudomonas sp. & $3(12)$ & $5(6)$ & \\
\hline Staphylococcus aureus & $1(4)$ & $6(7)$ & \\
\hline Streptococcus pneumoniae & $0(0)$ & $7(8)$ & \\
\hline Fungal & $0(0)$ & $0(0)$ & \\
\hline
\end{tabular}

The 32 patients with no infection had a median age of 65 (range $21-93) 31 \%(n=10)$ were female. They had a mean Charlson-score of 4.0 (range 0-9), a median delta-SOFA score of 3.0 (range 0-9), a 28-day mortality of $3 \%$, and direct transferral to ICU/HDU was noted in 10 cases $(31 \%)$. The 32 patients without infection eventually received the following discharge diagnoses: ketoacidosis $(n=5)$, dehydration $(n=4)$, syncope $(n=3)$, pulmonary embolism $(n=3)$, central chest pain $(n=3)$, exacerbation of chronic obstructive pulmonary disease $(n=2)$, intoxication $(n=2)$, stomach ache $(n=2)$, heart failure $(n=2)$, systemic inflammation $(n=2)$, peptic ulcer $(n=1)$, epilepsy $(n=1)$, drug fever $(n=1)$, head ache $(n=1)$, and stress $(n=1)$.

\section{Performance of calprotectin and other biomarkers}


The 367 sepsis alert patients in the study had the following median result: p-calprotectin 2.2 (IQR 1.13.8); CRP 67 (IQR 23-146); PTC 0.6 (IQR 0.2-6.0) NLR 11.4 (IQR 6.1-18.7). Figure 2 (A-D) shows the distribution of calprotectin, CRP, PTC and NLR in patients with infection with and without direct transferral to ICU/HDU, and patients without infection. Patients with infection and direct transferral to ICU/HDU had significantly higher $p$-calprotectin than patients with infection without direct transferral. No similar tendency was noted for PCT, CRP, or NLR. Figure 3 (A-D) shows the distribution of calprotectin, CRP, PTC and NLR among patients with bacterial infection, viral infection, and no infection. There was no significant difference for any of the biomarkers between the group with bacterial and viral infection. Only calprotectin could significantly distinguish between bacterial infection and no infection.

Table 2 shows median calprotectin levels for different patient characteristics. The median p-calprotectin values were similar between sex, age groups, source of infection and blood cultures with or without bacterial growth. Calprotectin was significantly higher in the two groups with the highest delta-SOFAvalues. 
Table 2

Calprotectin-values for patients with assumed infection for different patient characteristics.

\begin{tabular}{|c|c|c|}
\hline \multicolumn{3}{|c|}{ Calprotectin for patient with assumed infection (median and IQR) } \\
\hline & Calprotecin & $p$-value \\
\hline \multicolumn{3}{|l|}{ Sex } \\
\hline Female $(n=132)$ & $2.0(1.1-3.6)$ & \\
\hline Male $(n=203)$ & $2.4(1.3-3.9)$ & 0.3 \\
\hline \multicolumn{3}{|l|}{ Age, years } \\
\hline$<40(n=15)$ & $2.1(0.8-3.7)$ & \\
\hline $41-65(n=93)$ & $2.3(1.3-4.0)$ & 0.7 \\
\hline $66-80(n=139)$ & $2.1(1.2-3.5)$ & \\
\hline$>81$ & $2.4(1.3-3.9)$ & 0.3 \\
\hline \multicolumn{3}{|l|}{ Delta-SOFA } \\
\hline $0-1(n=58)$ & $1.8(1.1-3.5)$ & \\
\hline$>2-4(n=217)$ & $2.4(1.3-3.9)$ & 0.07 \\
\hline$>4(n=60)$ & $2.1(1.1-3.9)$ & 0.3 \\
\hline \multicolumn{3}{|l|}{ Sours of infection } \\
\hline Lower resp. $(n=127)$ & $2.6(1.5-4.3)$ & \\
\hline UTI $(n=72)$ & $2.2(1.3-3.9)$ & \\
\hline Abdomen $(n=36)$ & $1.7(0.9-3.3)$ & \\
\hline Skin $(n=41)$ & $2.3(1.2-3.7)$ & \\
\hline \multicolumn{3}{|l|}{ Co-morbidity (Charlson-score) } \\
\hline $0-1(n=22)$ & $2.5(1.3-5.8)$ & \\
\hline $2-5(n=169)$ & $2.1(1.1-3.9)$ & 0.2 \\
\hline $6-10(n=125)$ & $2.1(1.2-3.6)$ & \\
\hline$>10(n=19)$ & $3.0(1.8-5.9)$ & 0.06 \\
\hline $\operatorname{Sepsis}(n=277)$ & $2.3(1.3-3.9)$ & \\
\hline No sepsis $(n=58)$ & $1.8(1.1-3.5)$ & 0.1 \\
\hline Positive blood culture (117) & $2.1(1.2-3.9)$ & \\
\hline
\end{tabular}




\section{Calprotectin for patient with assumed infection (median and IQR)}

Negative blood culture (218) $\quad 2.3(1.2-3.8) \quad 0.6$

Figure 4 shows analysis of area under the curve (AUC) for need of ICU/ HDU care. When the analysis included all sepsis alert patients (Fig. 4A) and all patients with infections (Fig. 4B), p-calprotectin had significantly larger AUC than PTC and NLR and tended to have larger AUC than CRP. AUC-differences between calprotectin and the other biomarkers are shown in Table 3. As noted, AUC was significantly higher for calprotectin than the other biomarkers, both regarding all sepsis alert cases and regarding the patients with infection.

Table 3

Differences of areas under the receiver operating characteristics curves between p-calprotectin and CRP, PTC, and NLR, respectively.

\begin{tabular}{|llll|}
\hline \multicolumn{4}{|c|}{ All patients } \\
& AUC difference & $95 \% \mathrm{Cl}$ & p-value \\
\hline Calprotectin vs CRP & 0.119 & $0.222-0.014$ & 0.027 \\
\hline Calprotectin vs PTC & 0,205 & $0.327-0.113$ & 0.000 \\
\hline Calprotectin vs NLR & 0.186 & $0.288-0.084$ & 0.000 \\
\hline & Patients with infection & \\
\hline & AUC difference & $95 \%$ Cl & $p$-value \\
\hline Calprotectin vs CRP & 0.115 & $0.208-0.004$ & 0.049 \\
\hline Calprotectin vs PTC & 0.197 & $0.322-0.081$ & 0.001 \\
\hline Calprotectin vs NLR & 0.195 & $0.291-0.089$ & 0.000 \\
\hline
\end{tabular}

Table 4 shows sensitivities, specificities and predictive values for different p-calprotectin concentrations for patients transferred immediately to ICU/ HDU. 
Table 4

Diagnostic performance of $p$-calprotectin for discrimination between infected patients transferred to ICU/ HDU or ward.

\begin{tabular}{|lllll|}
\hline \multicolumn{4}{|l|}{ Sensitivity/ specificity for calprotectin if transferred to ICU/ HDU from ER* } \\
\hline Cut off value & Sensitivity (\%)** & Specificity (\%)*** & PPV**** & NPV***** \\
\hline 2 & $70(46 / 66)$ & $48(130 / 269)$ & $25(46 / 185)$ & $86.5(130 / 150)$ \\
\hline 2,5 & $60.5(40 / 66)$ & $60(163 / 269)$ & $27(40 / 146)$ & $86(163 / 189)$ \\
\hline 3 & $53(35 / 66)$ & $69.5(187 / 269)$ & $30(35 / 117)$ & $86(187 / 218)$ \\
\hline 3,5 & $48.5(32 / 66)$ & $76(204 / 269)$ & $33(32 / 97)$ & $86(204 / 238)$ \\
\hline 4 & $42.5(28 / 66)$ & $83(223 / 269)$ & $38(28 / 74)$ & $85(223 / 261)$ \\
\hline 4,5 & $38(25 / 66)$ & $84(226 / 269)$ & $37(25 / 68)$ & $85(226 / 267)$ \\
\hline$*$ Transferred to ICU/ HDU ( $n=66)$ all infected ( $n=335)$ & & \\
\hline$* *$ Sensitivity (\% \{no of true-positive cases/ all cases transferred to ICU/HDU\}) \\
\hline$* * *$ Specificity (\% \{no true-negative cases/ all cases transferred to ward\}) \\
\hline$* * *$ Positive predictive value (\% \{no true-positive cases/all cases above cut-off\}) \\
\hline$* * * *$ Negative predictive value (\% \{no true-negative cases/ all cases under cut-off\}) \\
\hline
\end{tabular}

Figure 5 shows changes in p-calprotectin, CRP, PCT, and NLR over time in for patients with repeated samples collected. Calprotectin was almost unchanged day 0 to day $2-3$, and after that decreasing. CRP and PTC continued to rise until day 5-7. NLR was decreasing from day 2-3.

\section{Discussion}

In the present study of sepsis alert patients, we found that the novel biomarker p-calprotectin was elevated in patients with established infection and was superior to CRP, PCT, and NLR for detecting patients in need of ICU or HDU care.

Calprotectin has been shown to be a potential biomarker for discriminating sepsis patients from other patients with infection (16-19). Due to the heterogeneity of sepsis patients, some researchers have focused on the role of calprotectin in multi-organ failure (27). We wanted to study calprotectin in a well characterized cohort of patients with clinical suspicion of sepsis already in the ED and evaluate whether calprotectin may be of value in the early assessment of sepsis. We found that patients with infection who the multidisciplinary team in the ED decided to transfer to the ICU or HDU had significantly higher calprotectin than other patients (Fig. 2). This was not explained by higher comorbidity (as measured by Charlson comorbidity score) or disease severity (as measured by delta-SOFA) on hospital arrival. There was no significant difference in delta-SOFA score between the group ending up direct in ICU or HDU and 
the group transferred to ward. This shows the difficulties in discrimination patients with sepsis so severe that they need ICU care by only using delta-SOFA score. In this case the patients were also assessed by three different doctors. This also shows the need for better biomarkers, where calprotectin could have a place.

CRP, PTC, and NLR could not discriminate between those who were admitted to ICU/HDU and those admitted to wards, in line with findings from similar studies $(10,18,27,28)$. ROC curve analysis thus showed that calprotectin was superior to CRP, PCT, and NLR in assessing the need for ICU/ HDU care (Fig. 4, Table 4).

As shown in Fig. 4, p-calprotectin had an AUC of 0.65 for predicting the for ICU/ HDU care. Larsson et al. recently studied p-calprotectin in ICU patients and found an AUC of 0.67 for discrimination between sepsis and non-sepsis in ICU, and 0.64 for prediction of ICU death, i.e. similar findings as in our study (20). However, when looking at sensitivities, specificities, and predictive values, we considered pcalprotectin $4.0 \mathrm{mg} / \mathrm{L}$ to be the best cut-off value for predicting the need for ICU/ HDU care, with a sensitivity of $42 \%$ and a specificity of $83 \%$. Larsson et al. found the best p-calprotectin cut-off to be 1.3 $\mathrm{mg} / \mathrm{L}$ for discrimination between sepsis and non-sepsis, with sensitivity $81 \%$ and specificity $56 \%$, and 1.1 $\mathrm{mg} / \mathrm{L}$ for prediction of ICU death, with sensitivity $80 \%$ and specificity $46 \%(20)$. Other studies have shown optimal calprotectin cut-offs of $3.4 \mathrm{mg} / \mathrm{L}$ for differentiating sepsis from post-operation inflammation (PPV47\% and NPV 69\%), e.g. in a study by Simm et al, and 2.2 (unknown unit) with sensitivity $62.5 \%$ and specificity $69.7 \%$ for blood culture positive sepsis in children in a study by Decambrinos et al. $(16,19)$. This might be related to patient selection, analytic method for $p$-calprotectin or the nature of the study question.

P-calprotectin and the other biomarkers did not differ considerably in performance based on sex, age, comorbidity and source of infection and there was no clear difference in p-calprotectin levels between bacterial and viral infection. This was also the case for CRP, PCT, and NLR, and may be due to the small number of patients with viral infection in the present study. Also, to be noted, p-calprotectin was not higher among patients with verified bacteremia (positive blood culture), compared to those with negative blood cultures in our study (Table 2).

Faecal calprotectin has been shown to be affected by immune suppression (29), but we find no difference in p-calprotectin levels in patients with or without immunosuppression.

On repeated samples, mostly NLR but also calprotectin decreased on day $2-3$, whereas CRP and PTC increased, and had started to decrease on day 5-7. This may reflect different inherent kinetics of the biomarkers. Our findings differ from those of Bartakova et al, and Simm et al $(16,18)$. In Bartakova's study all biomarkers hade the same dynamics as we found for calprotectin and NLR (18). However, in the study by Simm et al., only CRP had the same pattern as p-calprotectin (16). This may reflect different timing of sampling and a more heterogenous group of patients in our study compared with the others. 
Since calprotectin increases early during infection, it could be potentially be useful in the pre-hospital care setting. Further studies will be needed to define its exact role.

The present study has several strengths. First, our study cohort was well characterized and already clinically stratified for suspected sepsis, since it was part of a sepsis alert used in routine practice in the ED. All patients, even those who did not have any infection, could be assumed to be severely ill due to the inclusion criteria in the alert.

The study also has some limitations. For ethical reasons we did not try to obtain consent from relatives to patients who were already deceased. This was a concern for the 35 patients who died before consents were obtained, and we did not study how many of the deceased patients who were transferred to ICU or HDU before death. Thus, many severely ill patients were not included in the study and it is hard to assess how this might have affected the outcome of the study.

In conclusion, in sepsis alert patients, p-calprotectin was elevated in those who a multidisciplinary team decided to transfer immediately to the ICU/ HDU. P-calprotectin was superior to traditional biomarkers for predicting the need for ICU/ HDU care.

\section{Conclusion}

P-Calprotectin was found to be superior to CRP, PCT, and NLR for identification of patients with suspected sepsis who were in need of immediate ICU or HDU care. Thus, P-Calprotectin appears to be a useful biomarker in the management of sepsis.

the capacity to do that. P-calprotectin could there for have a place in the emergency when working with sever sick patients.

\section{Abbreviations}

PCT- procalcitonin

CRP- C-reactive protein

NLR- neutrophil-lymphocyte ratio

ICU- intensive care unit,

ED- emergency department

HDU- high dependent unit

SOFA- sequential organ failure assessment

AUC- area under the curve 


\section{Declarations}

Ethics: The study was approved by the Regional Ethical Committee in Stockholm (reference number 2017/1358-31).

Consent for publication: Not applicable

Availability of data: The dataset used and analyzed during the current study are available from the corresponding author on reasonable request.

There are no competing interests.

Funding: The study was funded by Gentian. It was supported by the Center for Innovative Medicine (CIMED) and Region Stockholm (ALF; 20180058), Swedish Governmental Agency for Innovation Systems (VINNOVA) under the frame of NordForsk (Project no. 90456, PerAID), and the Swedish Research Council under the frame of ERA PerMed (Project 2018-151, PerMIT).

All authors contributed equal to the study.

Acknowledgment: We thanks Gaetano Marrone for statistical aid and Aleksandra Havelka for critical review of the manuscript.

\section{References}

1. Mellhammar L, Wullt S, Lindberg A, Lanbeck P, Christensson B, Linder A. Sepsis Incidence: A Population-Based Study. Open Forum Infect Dis. 2016;3:207.

2. Deutschman CS, Tracey KJ. Sepsis: current dogma and new perspectives. Immunity. 2014;40:46375.

3. Fleischmann C, Scherag A, Adhikari NK, Hartog CS, Tsaganos T, Schlattmann P, et al. Assessment of Global Incidence and Mortality of Hospital-treated Sepsis. Current Estimates and Limitations. Am J Respir Crit Care Med. 2016;193:259-72.

4. Rhee C, Dantes R, Epstein L, Murphy DJ, Seymour CW, Iwashyna TJ, et al. Incidence and Trends of Sepsis in US Hospitals Using Clinical vs Claims Data, 2009-2014. JAMA. 2017;318:1241-9.

5. Rudd KE, Johnson SC, Agesa KM, Shackelford KA, Tsoi D, Kievlan DR, et al. Global, regional, and national sepsis incidence and mortality, 1990-2017: analysis for the Global Burden of Disease Study. Lancet. 2020;395:200-11.

6. Reinhart KDR, Kissoon N, Machado FR, Schachter RD, Finfer S. Recognizing S epsis as a Global Health Priority -A WHO Resolution. N Engl J Med. 2017;377:414-7.

7. Levy MM, Gesten FC, Phillips GS, Terry KM, Seymour CW, Prescott HC, et al. Mortality Changes Associated with Mandated Public Reporting for Sepsis. The Results of the New York State Initiative. Am J Respir Crit Care Med. 2018;198:1406-12. 
8. Viale P, Tedeschi S, Scudeller L, Attard L, Badia L, Bartoletti M, et al. Infectious Diseases Team for the Early Management of Severe Sepsis and Septic Shock in the Emergency Department. Clin Infect Dis. 2017;65:1253-9.

9. Coopersmith CM, De Backer D, Deutschman CS, Ferrer R, Lat I, Machado FR, et al. Surviving sepsis campaign: research priorities for sepsis and septic shock. Intensive Care Med. 2018;44:1400-26.

10. Hamade B, Huang DT. Procalcitonin: Where Are We Now? Crit Care Clin. 2020;36:23-40.

11. Hotchkiss RS, Monneret G, Payen D. Sepsis-induced immunosuppression: from cellular dysfunctions to immunotherapy. Nat Rev Immunol. 2013;13:862-74.

12. Huang Z, Fu Z, Huang W, Huang K. Prognostic value of neutrophil-to-l ymphocyte ratio in sepsis: A meta-analysis. Am J Emerg Med. 2019.

13. de Jager CP, van Wijk PT, Mathoera RB, de Jongh-Leuvenink J, van der Poll T, Wever PC.

Lymphocytopenia and neutrophil-lymphocyte count ratio predict bacteremia better than conventional infection markers in an emergency care unit. Crit Care. 2010;14:192.

14. Lipcsey M, Hanslin K, Stalberg J, Smekal D, Larsson A. The time course of calprotectin liberation from human neutrophil granulocytes after Escherichia coli and endotoxin challenge. Innate Immun. 2019;25:369-73.

15. Huang L, Li J, Han Y, Zhao S, Zheng Y, Sui F, et al. Serum Calprotectin Expression as a Diagnostic Marker for Sepsis in Postoperative Intensive Care Unit Patients. J Interferon Cytokine Res. 2016;36:607-16.

16. Simm M, Soderberg E, Larsson A, Castegren M, Nilsen T, Eriksson M, et al. Performance of plasma calprotectin as a biomarker of early sepsis: a pilot study. Biomark Med. 2016;10:811-8.

17. Jonsson N, Nilsen T, Gille-Johnson P, Bell M, Martling CR, Larsson A, et al. Calprotectin as an early biomarker of bacterial infections in critically ill patients: an exploratory cohort assessment. Crit Care Resusc. 2017;19:205-13.

18. Bartakova E, Stefan M, Stranikova A, Pospisilova L, Arientova S, Beran O, et al. Calprotectin and calgranulin C serum levels in bacterial sepsis. Diagn Microbiol Infect Dis. 2019;93:219-26.

19. Decembrino L, De Amici M, Pozzi M, De Silvestri A, Stronati M. Serum Calprotectin: A Potential Biomarker for Neonatal Sepsis. J Immunol Res. 2015;2015:147973.

20. Larsson A, Tyden J, Johansson J, Lipcsey M, Bergquist M, Kultima K, et al. Calprotectin is superior to procalcitonin as a sepsis marker and predictor of 30-day mortality in intensive care patients. Scand $\mathrm{J}$ Clin Lab Invest. 2019:1-6.

21. Silvin A, Chapuis N, Dunsmore G, Goubet AG, Dubuisson A, Derosa L, et al. Elevated Calprotectin and Abnormal Myeloid Cell Subsets Discriminate Severe from Mild COVID-19. Cell. 2020;182:1401-18 e18.

22. Ljunggren $M$, Castren $M$, Nordberg M, Kurland L. The association between vital signs and mortality in a retrospective cohort study of an unselected emergency department population. Scand J Trauma Resusc Emerg Med. 2016;24:21. 
23. Yu D, Larsson A, Parke A, Unge C, Henning C, Sunden-Cullberg J, et al. Single-Sampling Strategy vs. Multi-Sampling Strategy for Blood Cultures in Sepsis: A Prospective Non-inferiority Study. Front Microbiol. 2020;11:1639.

24. Seymour CW, Gesten F, Prescott HC, Friedrich ME, Iwashyna TJ, Phillips GS, et al. Time to Treatment and Mortality during Mandated Emergency Care for Sepsis. N Engl J Med. 2017;376:2235-44.

25. Kadri SS, Rhee C, Strich JR, Morales MK, Hohmann S, Menchaca J, et al. Estimating Ten-Year Trends in Septic Shock Incidence and Mortality in United States Academic Medical Centers Using Clinical Data. Chest. 2017;151:278-85.

26. Singer M, Deutschman CS, Seymour CW, Shankar-Hari M, Annane D, Bauer M, et al. The Third International Consensus Definitions for Sepsis and Septic Shock (Sepsis-3). JAMA. 2016;315:80110.

27. Fisher J, Linder A. Heparin-binding protein: a key player in the pathophysiology of organ dysfunction in sepsis. J Intern Med. 2017;281:562-74.

28. Lelubre C, Anselin S, Zouaoui Boudjeltia K, Biston P, Piagnerelli M. Interpretation of C-reactive protein concentrations in critically ill patients. Biomed Res Int. 2013;2013:124021.

29. Roseth AG, Aadland E, Grzyb K. Normalization of faecal calprotectin: a predictor of mucosal healing in patients with inflammatory bowel disease. Scand J Gastroenterol. 2004;39:1017-20.

\section{Figures}

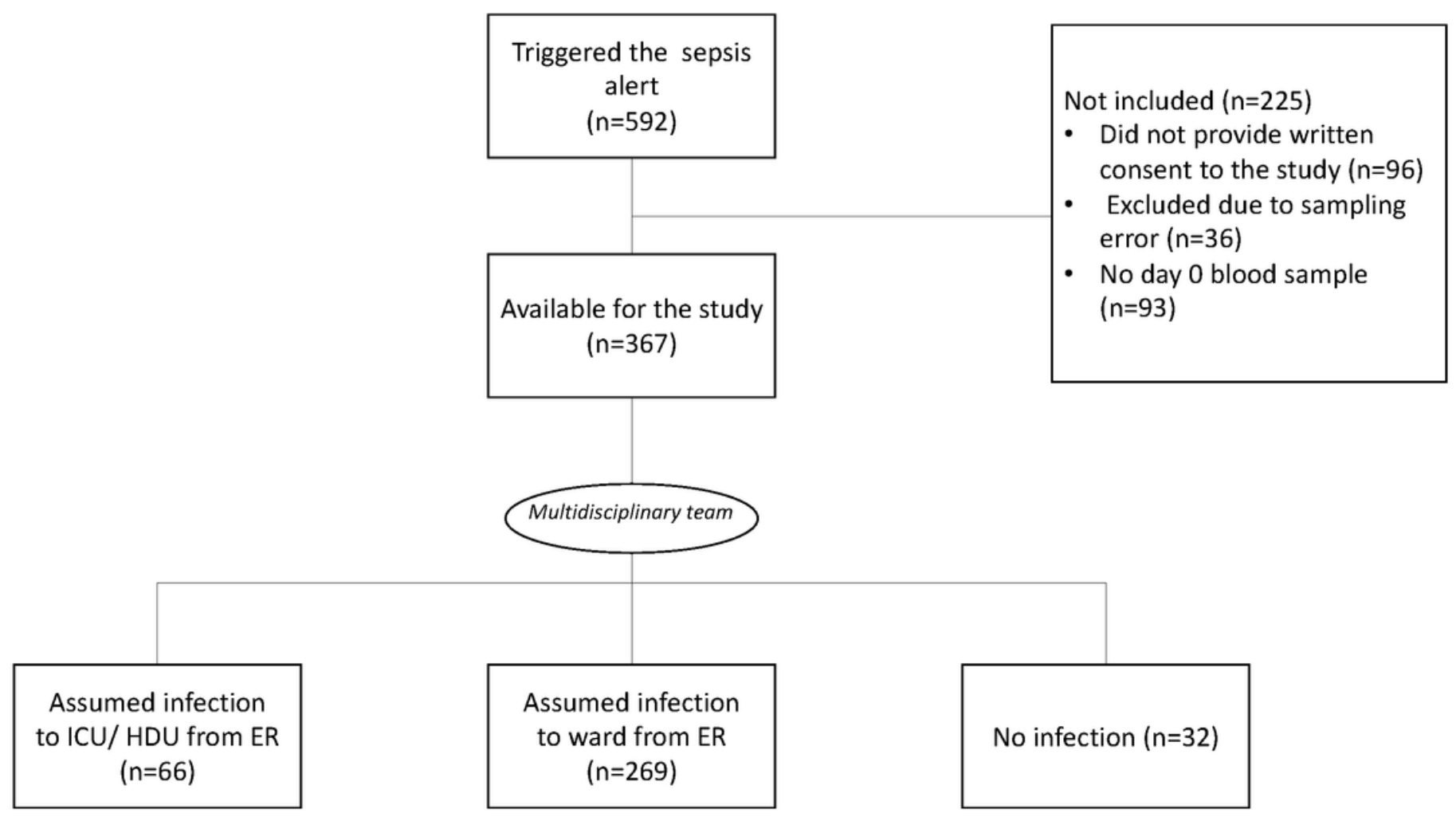


Patient selection flowchart.

Figure 2A

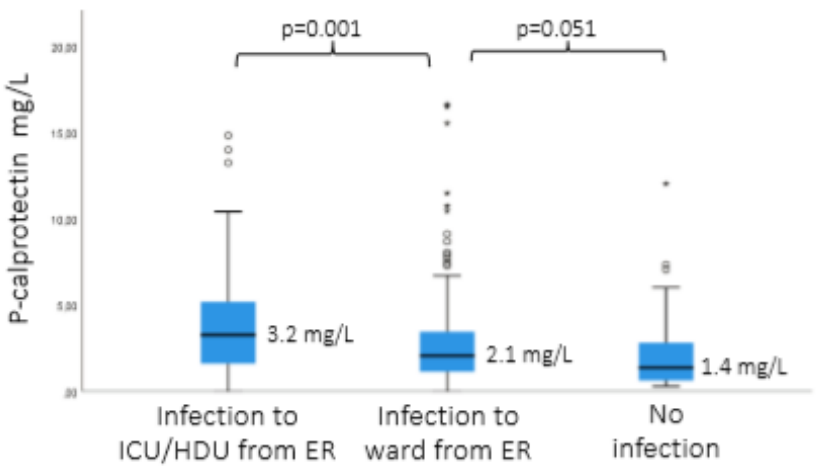

Figure 2C

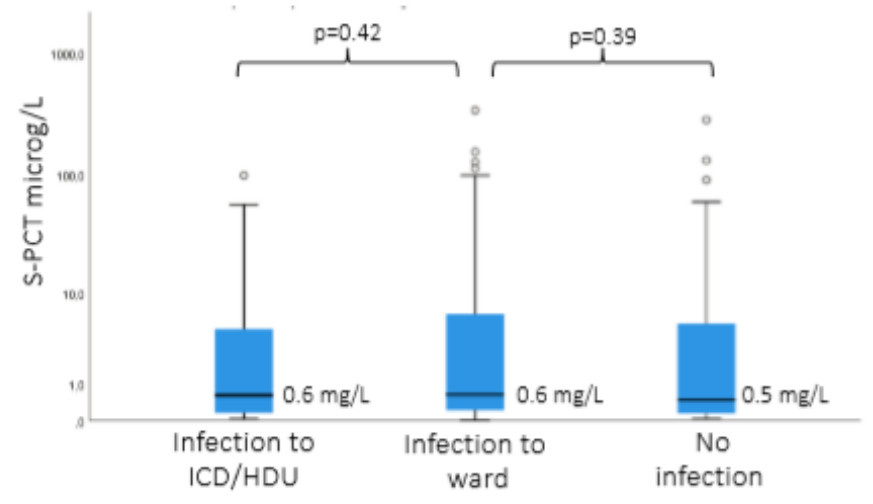

Figure 2B

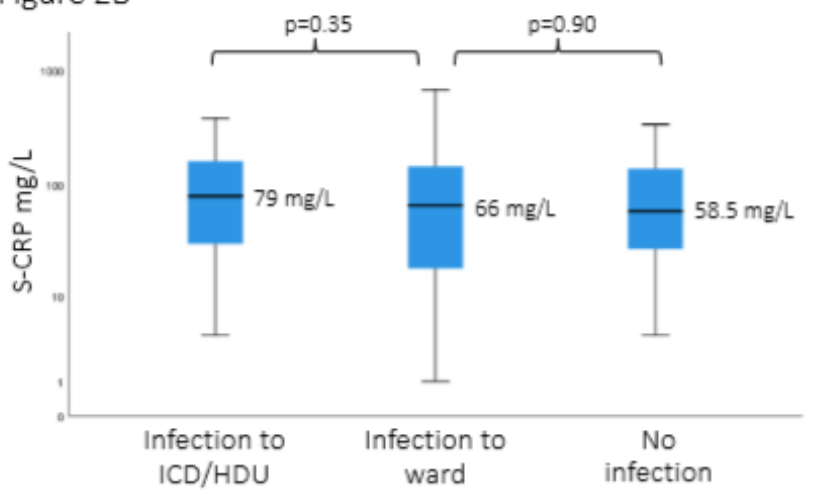

Figure 2D

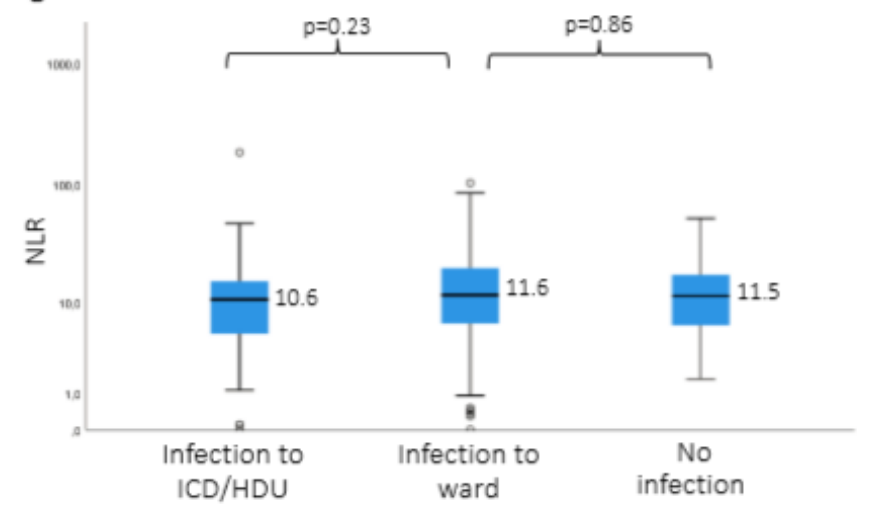

Figure 2

Comparison of p-calprotectin (A), CRP (B), PTC (C), and NLR (D) concentrations among all patients. 
Figure 3A

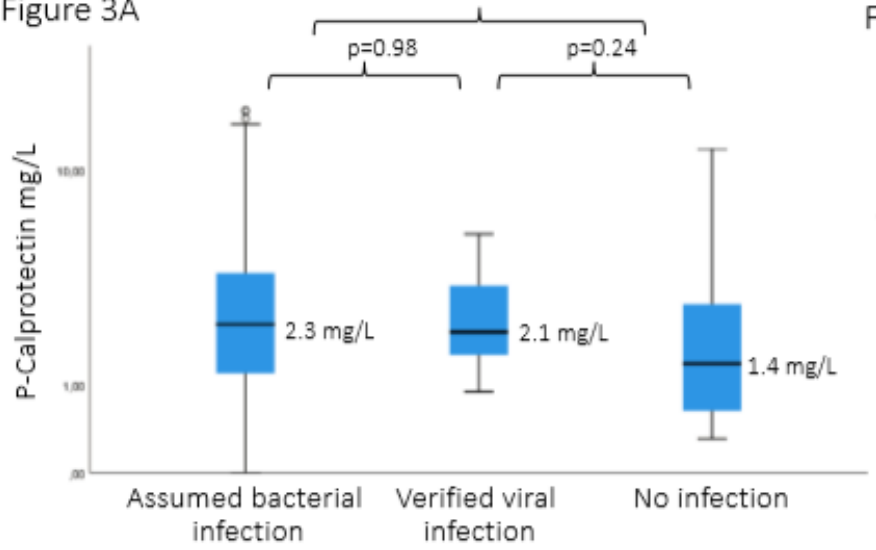

Figure $3 \mathrm{C}$

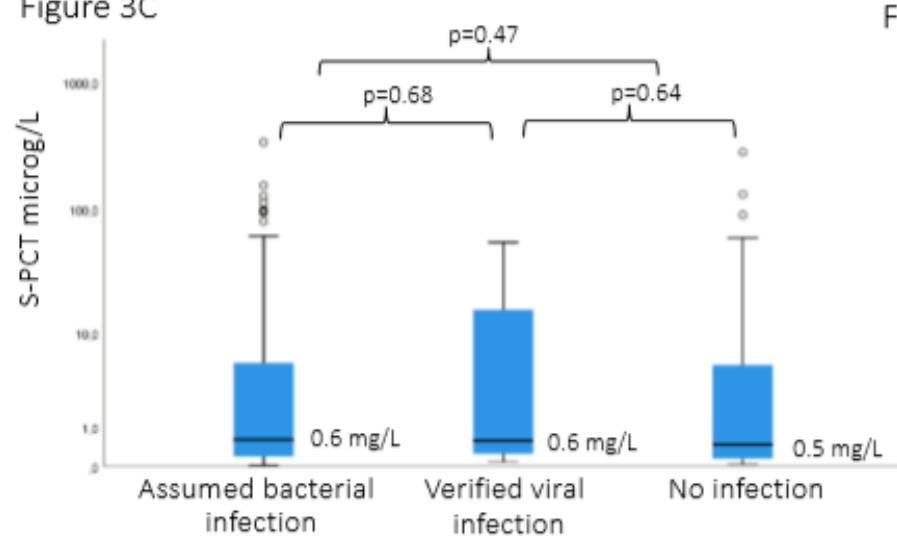

Figure 3B

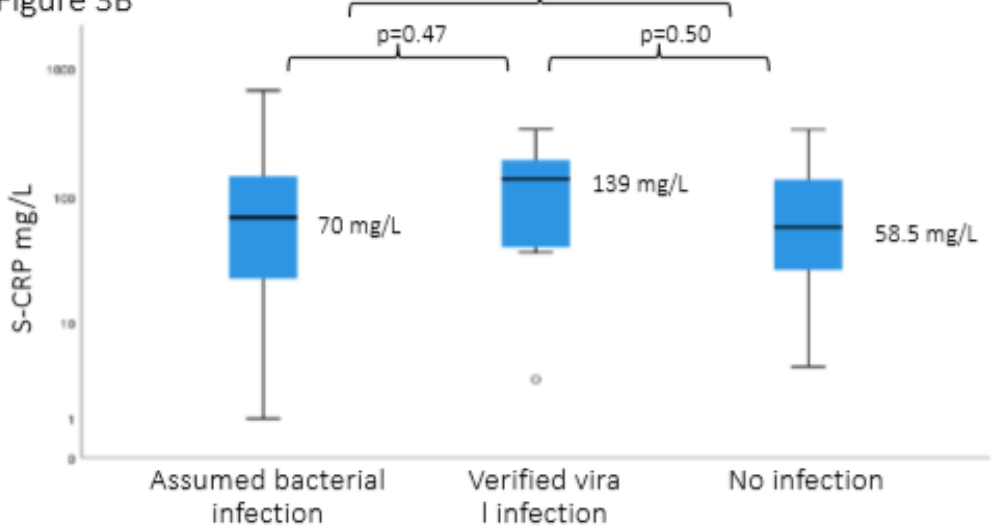

Figure 3D

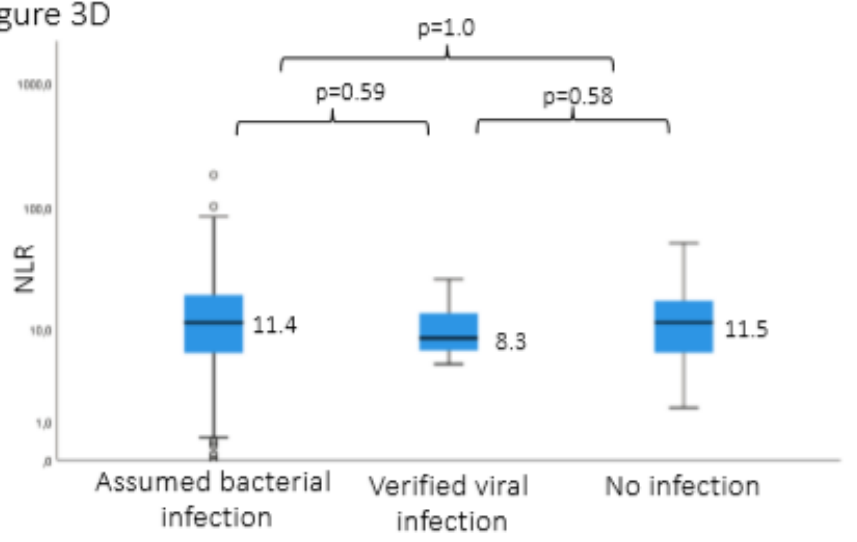

\section{Figure 3}

Comparison of plasma calprotectin (A), CRP (B), PTC (C) and NLR (D) concentrations among all patients.

Figure 4A All patients

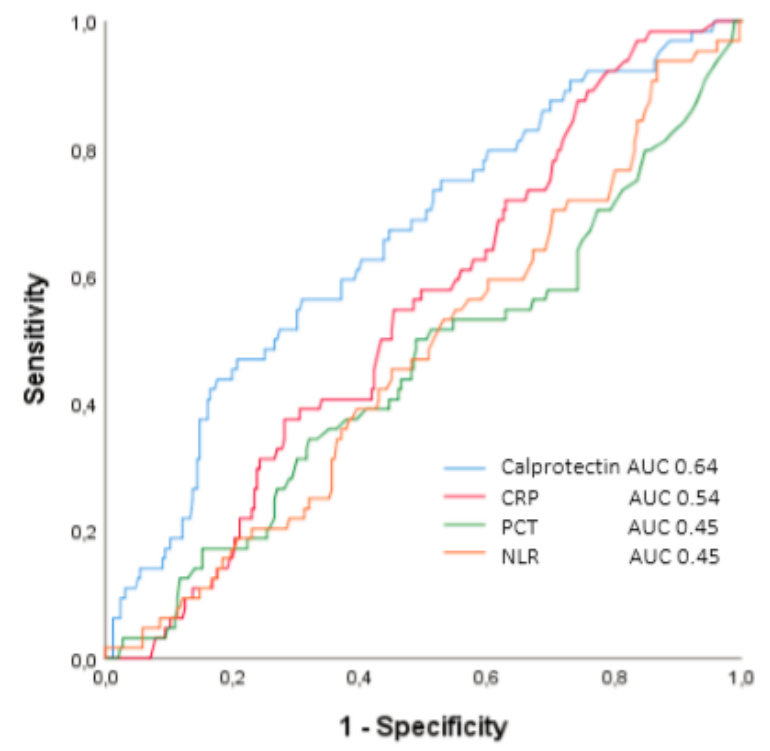

Figure $4 \mathrm{~B}$ Infected patients

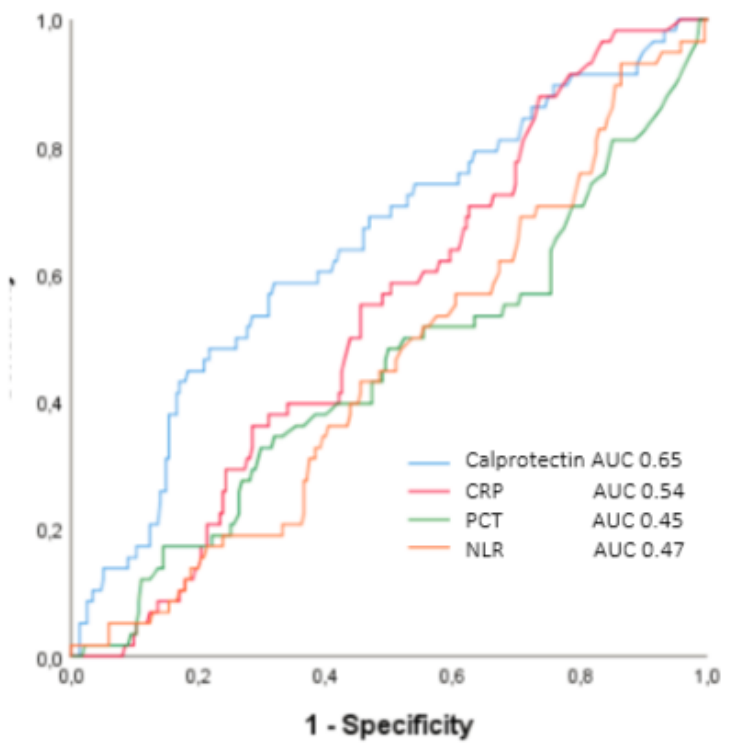

\section{Figure 4}

Receiver operating characteristics analysis of calprotectin, CRP, PTC and NLR. 
Figure 5A

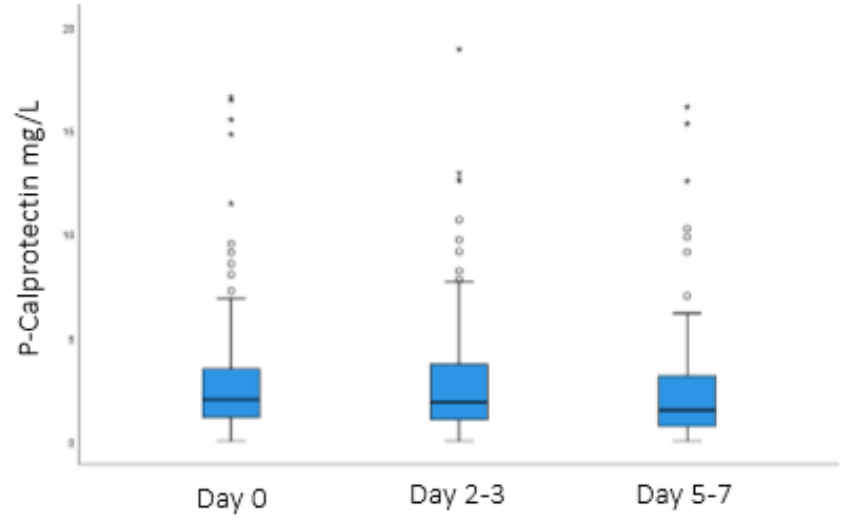

Figure $5 C$

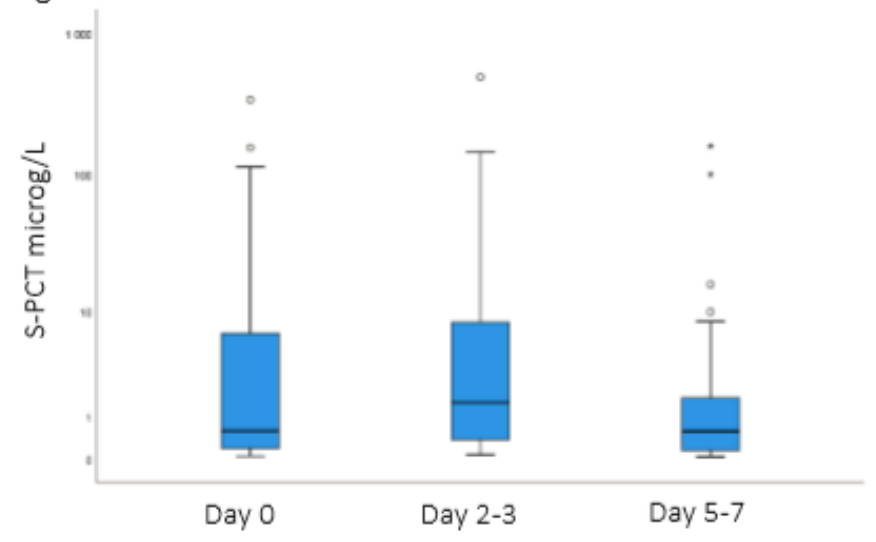

Figure 5B

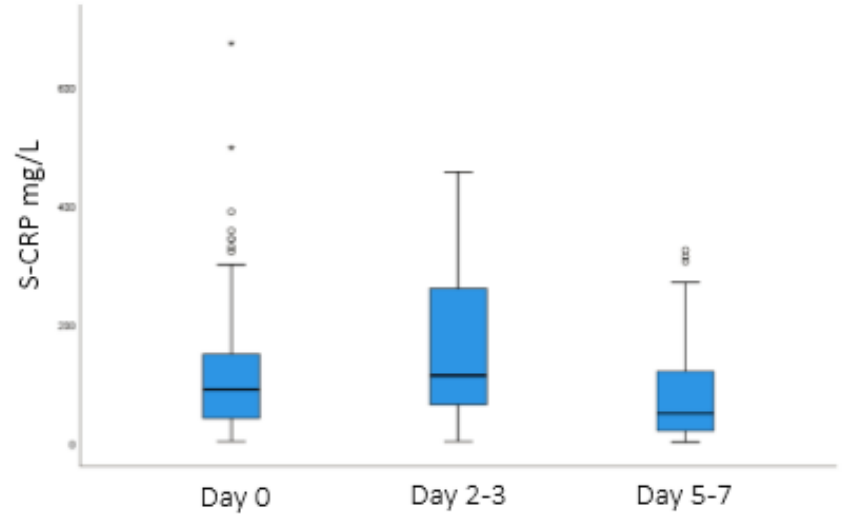

Figure 5D

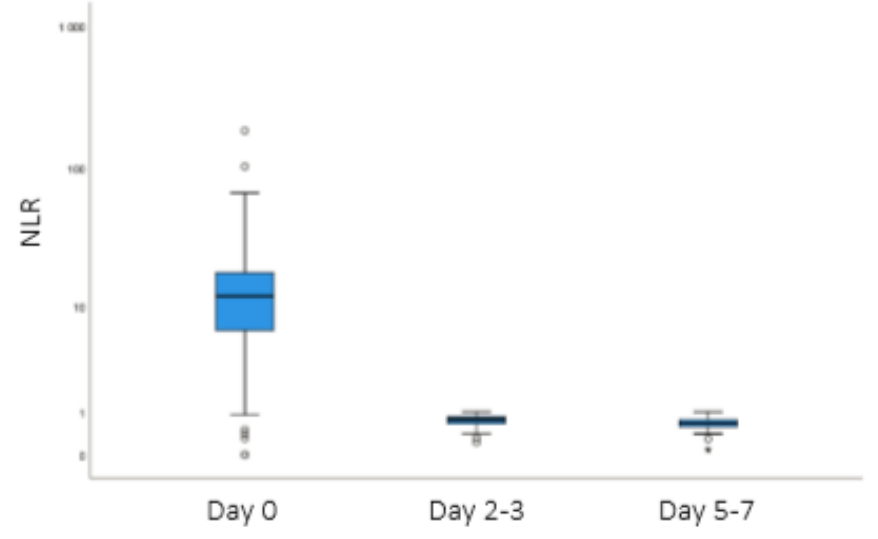

Figure 5

Kinetics of p-calprotectin (A), CRP (B), PTC (C) and NLR(D) during hospitalisation. 